\title{
Development of electrochemical sensor based on thiolated calixarene- functionalized gold nanoparticles for the selective recognition of anthracene
}

\begin{abstract}
In this work, an electrochemical sensor for anthracene detection was constructed based on a modified electrode consisting of thiolated calixarene (TC4)-functionalized gold nanoparticles (AuNPs) and electrochemically reduced graphene oxide (ERGO). Firstly, graphene oxide GO) was reduced on screen printed carbon electrodes (SPCE), followed by the immobilization of AuNPs and TC4 through a self-assembly process to form TC4/AuNPs/ ERGO-SPCE. The molecules' structure was characterized using FTIR and Raman spectroscopy, the sample's morphology and topology were characterized using Field Emission Scanning Electron Microscopy (FESEM), High Resolution Transmission Electron Microscopy (HRTEM) and electrochemical performance of developed sensor was characterized using electrochemical method. Owing to the outstanding supramolecular recognition of anthracene by TC4, the target analyte was detected accurately with a linear range of 1-7 $\mu \mathrm{M}$ and limit of detection down to $0.0625 \mu \mathrm{M}(\mathrm{S} / \mathrm{N}=3)$. Additionally, the sensor was acknowledged for its excellent antiinterference properties in the presence of organic compounds as well as inorganic metal ions. The proposed sensor also yielded comparable results to conventional HPLC method, with a satisfactory recovery of $92-98 \%$ in environmental samples.
\end{abstract}

Keyword: Anthracene; Electrochemically reduced graphene oxide; Gold nanoparticles; Thiolated-calixarene 